(C) 2018 IEEE. Personal use of this material is permitted. Permission from IEEE must be obtained for all other uses, in any current or future media, including reprinting/republishing this material for advertising or promotional purposes, creating new collective works, for resale or redistribution to servers or lists, or reuse of any copyrighted component of this work in other works. 


\title{
Static Var Compensator Allocation Considering Transient Stability, Voltage Profile and Losses
}

\author{
Sahand Ghavidel ${ }^{1 *}$, Ali Azizivahed ${ }^{2}$, Mostafa Barani ${ }^{2}$, Jamshid Aghaei ${ }^{2}, \mathrm{Li} \mathrm{Li}^{1}$, and Jiangfeng Zhang ${ }^{1}$ \\ ${ }^{1}$ Faculty of Engineering and Information Technology, University of Technology Sydney, PO Box 123, Broadway, NSW 2007, \\ Australia \\ ${ }^{2}$ Department of Electrical and Electronics Engineering, Shiraz University of Technology, Shiraz, Iran \\ 1*Email: sahand.ghavideljirsaraie@ student.uts.edu.au
}

\begin{abstract}
The purpose of this paper is to determine the optimal location, size and controller parameters of Static Var Compensator (SVC) to simultaneously improve static and dynamic objectives in a power system. Four goals are considered in this paper including transient stability, voltage profile, SVC investment cost and power loss reduction. Along with the SVC allocation for improving the system transient stability, an additional controller is used and adjusted to improve the SVC performance. Also, an estimated annual load profile including three load levels is utilized to accurately find the optimal location and capacity of SVC. By considering three load levels, the cost of power losses in the power system is decreased significantly. The combination of the active power loss cost and SVC investment cost is considered as a single objective to obtain an accurate and practical solution, while the improvement of transient stability and voltage profile of the system are considered as two separate objectives. The problem is therefore formulated as a multi-objective optimization problem, and Multi Objective Particle Swarm Optimization (MOPSO) algorithm is utilized to find the best solutions. The suggested technique is verified on a 10-generator 39-bus New England test system. The results of the nonlinear simulation indicate that the optimal sizing, location and controller parameters setting of SVC can improve significantly both static and dynamic performance of the system.
\end{abstract}

Index Terms-- Static Var Compensator (SVC), Transient stability, Multi Objective Particle Swarm Optimization (MOPSO), Voltage profile.

\section{INTRODUCTION}

\section{A. Aims and Scope}

In recent years, Flexible AC Transmission Systems (FACTS) devices have been utilized for various objectives to improve the power system operation [1]. The main objectives which are essential for the operation and security of power systems include: i) voltage profile, ii) power loss, and iii) transient stability [1]. Among the mentioned objectives, transient stability is an increasingly important issue in the power system, e.g., a weak transient stability may frequently cause the blackout during the system fault, and it can extremely damage the rotor of generators. In order to mitigate these difficulties, FACTS devices, which are fast responsive, can be utilized. In addition, FACTS devices can improve the voltage profile in the power system [2]. Electrical devices are designed to work within a specific range of voltage. Therefore, the deviation from this range reduces the efficiency of devices and can deteriorate their operation or even damage them. In this regard, FACTS devices can be used to provide voltage security constraints in the power systems under normal conditions. Consequently, the FACTS devices can improve the mentioned objectives in the power system. However, the effectiveness of the FACTS controllers is mainly dependent on their locations and capacity. Therefore, it is essential to propose practical method for determining the allocation and capacity of these devices in the power system.

\section{B. Literature Review and Approach}

A considerable amount of literature has been published to evaluate the impacts of FACTS devices in the power system and determine their optimal allocations. To this end, different criteria have been proposed in the literature for the allocation problem. For example, Ref. [3] considers the static voltage stability enhancement as an objective for the allocation problem. Loss reduction is the main criterion which is considered for the allocation problem in [4]. Power plants fuel cost reduction using optimal power flow and voltage profile improvement are the other objectives proposed in [2]. In order to cope with the small signal stability problem, Ref. [5] proposes the best assignment and parameter setting of FACTS devices. In [6], the Static Var Compensator (SVC) has been allocated to enhance the first swing stability boundary of the power system. In order to advance the transient stability of the system and SVC cost, the optimal location, size and setting parameters of SVC controller are evaluated in [7]. Also, Ref. [8] determines the optimal location, size and parameter setting of SVC in long transmission lines to improve transient stability of the system and reduce the SVC cost. It should be noted that each of the mentioned objectives improves the power system network operation, but improvement in one objective does not guarantee the same improvement in others.

In addition, some assumptions, e.g., using single objective optimization, ignoring the investment budget as a part of the objective function, and allocation in the presence of a multi-objective function [9], have been considered in the literature to implement these objectives. These assumptions can result in some problems such as, an inability to use the powerful advantages of FACTS devices in the static and dynamic conditions and impractical allocation results. Note that, each of the 
mentioned objectives can enhance the operation of the power system from its own viewpoint and therefore, none of them can be neglected for allocation of FACTS devices. Furthermore, It is essential to consider the cost of devices since neglecting it cannot be justified in the allocation of FACTS devices [7, 9]. The current paper considers the transient stability improvement, power loss reduction, voltage profile, and the investment costs of FACTS devices to improve previous researches in the field of FACTS devices allocation in the power systems. Despite previous studies, the alleviation of both cost factors is considered in the proposed model. In an effort to approach a practical solution, an estimated annual load profile has been considered. It should be mentioned that, in this study, the FACTS device is assumed to be SVC.

One additional controller is required, when a SVC is utilized to improve the voltage of buses in a power system. This kind of controller can be used to adjust the bus voltage of SVC to improve the damping procedure of the system oscillations [7-9]. In this situation, the interaction between the power system and this controller (SVC-based controller) can affect the system oscillations. Accordingly, the optimum parameter setting of this kind of controller is essential and it should be selected properly. A lot of approaches, for example stochastic exploration, have been proposed and advanced to find global optimization solutions $[10,11]$. In order to improve the system transient stability, this paper determines the optimal location of the SVC by considering and adjusting an extra controller to enhance its performance.

Considering more than one objective function increases complexity of the optimization model [12-14]. In order to solve this kind of problems, multi-objective optimization methods can be employed. In the MultiObjective Problem (MOP) unlike the single one, a set of solutions obtained instead of only one answer. In this paper, Pareto method has been used to solve the mentioned problem. The Pareto optimal solution is the solution that improvement in one of the objective function begins to deteriorate its performance in at least one of the rest. The Pareto method allows the system designer to choose among the available solutions with respect to the network's conditions and requirements for determining the placement and capacity of SVC. Due to the simple concept, easy implementation, modifiable parameters and rapid convergence, Multi-Objective Particle Swarm Optimization (MOPSO) algorithm has been utilized for solving various optimization problems [15-17]. In order to solve the mentioned MOP, this paper employs MOPSO as a promising evolutionary technique. In addition, a Sequential Quadratic Programming (SQP) optimization sub-problem has been utilized to implement an estimated annual load profile to accurately find the optimum location and capacity of SVC.

\section{Paper Organization}

The remainder of the paper is organized as follows. Section II formulates the optimal location and size of
SVC as a multi-objective optimization problem. Next, a brief overview of SVC-based controller is presented in Section III. Section VI provides results for a case study. Finally, Section V summarizes the results of this work and draws conclusions.

\section{PROBlem Formulation AND OBJECTIVE FunCTION}

The first objective function in this paper is related to minimization of the investment cost of SVC and active power loss. This objective function is as follows [7],

$$
\begin{aligned}
f_{1}(x, u, w) & =K_{i} C_{\text {investment }}(w) \\
& +K_{e} \sum_{i}\left(P_{\text {lossi }}(x, u, w) T_{i}\right)
\end{aligned}
$$

where $K_{e}$ is the active power cost in $\$ / \mathrm{kWh} ; T_{i}$ represents the time length of the $i^{t h}$ load level in hours; $P_{\text {lossi }}(x, u, w)$ is the active power loss of $i^{\text {th }}$ load level; $C_{\text {investment }}(w)$ can be written as follows [7]:

$$
C_{\text {investment }}(w)=C_{M \text { var_SVC }} S_{S V C}
$$

where $S_{S V C}$ represents the apparent power of SVC; $C_{M \text { var_SVC }}$ is the MVar cost of SVC [7].

$$
\begin{aligned}
C_{M \text { var_SVC }_{-}=} & 0.3 S_{S V C}^{2}-305 S_{S V C} \\
& +127380 \text { (\$/MVar) }
\end{aligned}
$$

Note that, the investment cost needs to be accomplished in the same year of the allocation study. After calculating the investment cost of SVC based on the interest rate, the life time of SVC can be combined in a single objective function. The following $K_{i}$ factor can be defined to do this [7].

$$
K_{i}=\frac{(1+B)^{n_{S V C}} B}{(1+B)^{n_{S V C}}-1}
$$

where $B$ presents the refundable investment rate in percentage; $n_{S V C}$ is the SVC life time. $B$ and $n_{S V C}$ are assumed to be 15 percent and 30 years, respectively.

The transient stability of the system is considered as the second objective function as follows [7].

$$
f_{2}(x, u, w)=\int_{0}^{t=t_{\text {sim }}}\left(\sum_{i=1}^{4}\left|J_{i}\right|\right) t d t,
$$

where $J_{i}$ are chosen as the maximum selected values of speed deviations from the set of $J^{k}$ as follow [7]:

$$
J^{k}=\int_{0}^{t=t_{\text {sim }}}\left(\sum_{i=1}^{N_{G}} \sum_{j=i+1}^{N_{G}}\left(\left|\Delta \omega_{i, j}{ }^{k}\right|\right)\right) t d t,
$$

where $\Delta \omega_{i, j}$ represents the speed deviation among generators $i$ and $j\left(\Delta \omega_{i}-\Delta \omega_{j}\right) ; N_{G}$ is the total number of generators in the system; $t_{\text {sim }}$ is the time of simulation horizon. The $J^{k}$ set is generated in case that there is no SVC in the system. As the Integral of Time multiple Absolute Error (ITAE) is used to derive the objective, the advantage of the minimal requirements of dynamic plant information can be preserved. Also, to compute this objective function, the time-domain simulation is used. The aim is minimizing the objective function $f_{2}$ to improve the overshoots and settling time of the response [7]. 
The third objective function is the voltage limitations and violations in the system. The voltage violation can be defined as follows for each bus.

$$
V D_{i}=\frac{\Phi\left(\left|V_{i}-V_{i}^{\text {ideal }}\right|-d v_{i}\right)}{v_{i}}, \Phi(x)= \begin{cases}0 & \text { if } x<0 \\ x & \text { otherwise }\end{cases}
$$

where $V_{i}, V_{i}^{\text {ideal }}$ are the voltage and ideal voltage (i.e. 1 $\mathrm{pu}) ; d v_{i}$ represents the maximum voltage deviation tolerance. Accordingly, the third objective function can be written as follows.

$$
f_{3}(x, u, w)=\sum_{i \in J_{L}} V D_{i}=\sum_{i \in J_{L}} \frac{\Phi\left(\left|V_{i}-V_{i}^{\text {ideal }}\right|-d v_{i}\right)}{v_{i}}
$$

where $J_{L}$ is the number of buses. Note that, by minimizing this objective function, the bus voltages will remain in the specified limits.

To solve the multi-objective optimization problem, some constraints such as the bound of location, capacity of SVC and limits of the controller parameters have been considered. Therefore, the multi-objective optimization problem can be presented as follows:

$$
\begin{gathered}
\min _{u, w \in \chi} f_{1}(x, u, w) \\
\min _{u, w \in \chi} f_{2}(x, u, w) \\
\min _{u, w \in \chi} f_{3}(x, u, w)
\end{gathered}
$$

Subject to

$$
\begin{gathered}
N_{l o c}^{\min } \leq N_{l o c} \leq N_{l o c}^{\max } \\
B_{S V C}^{\min } \leq B_{S V C} \leq B_{S V C}^{\max } \\
K_{S}^{\min } \leq K_{S} \leq K_{S}^{\max } \\
T_{1 S}^{\min } \leq T_{1 S} \leq T_{1 S}^{\max } \\
T_{3 S}^{\min } \leq T_{3 S} \leq T_{3 S}^{\max }
\end{gathered}
$$

where $B_{S V C}$ and $N_{l o c}$ are the capacity and location number the SVC, respectively. $K_{S}, T_{I S}, T_{2 S}$ are the SVC controller parameters. The MOPSO technique is taken from [8] to solve the multi-objective optimization problem in this paper.

\section{SVC-BASED CONTROLLER}

The structure of the SVC-based controller is shown in Fig. 1. As it can be seen, the common lead-lag structure with gain, washout and two-stage phase-compensation blocks is used.

The washout block, which is a high-pass filter, is used to allow the passing of oscillations in the input signal without variation. This block cannot affect the steady changes in the input. The washout time constant can have a range between 1 to 20 seconds [18]. To provide the phase-lead behavior to compensate the phase-lag between input and output signals, the phase-compensation block is used.

TABLE I

INFORMATION FOR ECONOMIC STUDY

\begin{tabular}{c|c}
\hline \hline Parameter & Values \\
\hline Factor and duration of load level 1 & $0.81,2136$ hours \\
Factor and duration of load level 2 & $1.00,2832$ hours \\
Factor and duration of load level 3 & $0.90,3792$ hours \\
\hline
\end{tabular}

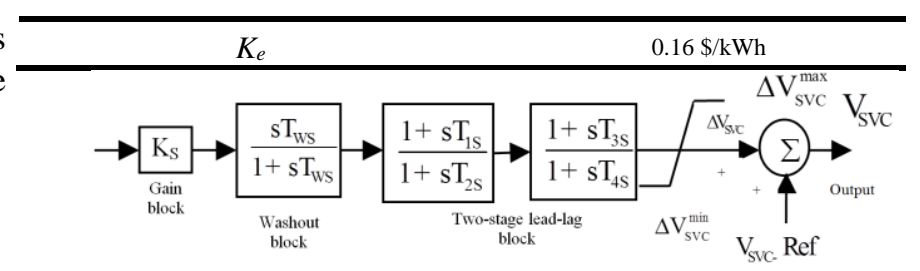

Fig. 1 SVC-based controller.

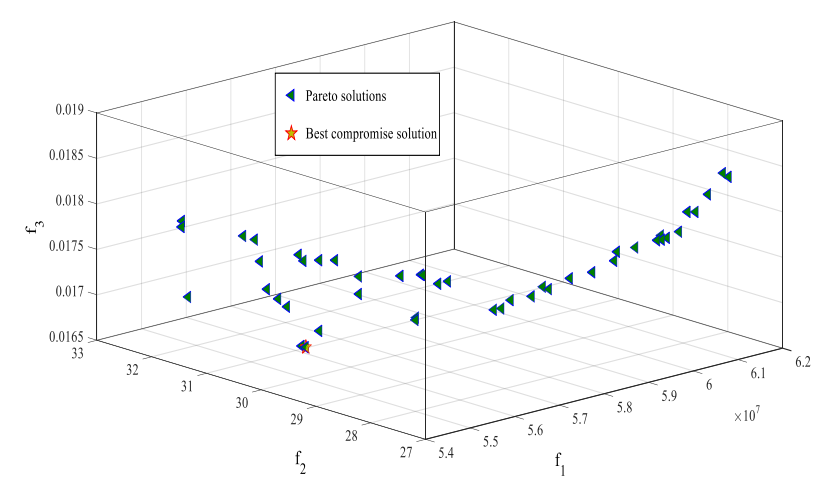

Fig. 2 Non-dominated and the finest cooperation answers.

Generally, in the SVC-based controller structure the time constants need to be pre-specified. In this paper, $T_{W}=10 \mathrm{~s}$ and $T_{2 S}=T_{4 S}=0.3 \mathrm{~s}$ are assumed. To determine the time constants $T_{I S}, T_{3 S}$ and the gain $K_{S}$, the MOPSO technique is used.

\section{RESUlTS AND DISCUSSIONS}

The 10-machine 39-bus New England power system is utilized to define the optimum location and size of SVC and determine the parameters of the SVC-based controller [7, 19]. Generator 1 (bus 39) represents parts of the U.S.Canadian interconnection system [7]. It is expected here that SVC can be installed at all buses excepting bus 39 . Table I lists the necessary information for economic study, and the forecasted load curve with three load levels and their durations. The fault is set to happen at $2.0 \mathrm{~s}$ from the beginning of the simulation and be cleared after $1.0 \mathrm{~s}$ at bus 29 at the end of line 26-29, which is enormously severe from the stability viewpoint $[7,20]$.

The subsequent objective function is recommended to calculate the transient stability of the system [7]:

$$
f_{2}(x, u, w)=\int_{0}^{t=t_{\text {sim }}}\left(\left|J_{1}\right|+\left|J_{2}\right|+\left|J_{3}\right|+\left|J_{4}\right|\right) t d t,
$$

where $j_{1}=\Delta \omega_{4}-\Delta \omega_{10}, j_{2}=\Delta \omega_{6}-\Delta \omega_{10}, j_{3}=\Delta \omega_{7}-\Delta \omega_{10}$ and $j_{4}=\Delta \omega_{8}-\Delta \omega_{10}$. The voltage magnitude of the buses should vary in the band between 0.97 and $1.03 \mathrm{pu}$. The ranges of the optimized parameters are $0.01-10 p u$ for $B_{S V C}, 0.01-1$ for $T_{I S}$ and $T_{3 S}, 0.01-200$ for $K_{S}$ and all load bus numbers for $N_{l o c}$. In all MOPSO runs, the number of population is selected to be 100 and the maximum number of iterations is set to 50 [7].

Fig. 2 shows the non-dominated answers of optimum position, size and controller parameters of SVC that are obtained from MOPSO algorithm. Also, Tables II and III show the results acquired by MOPSO and the best compromise solution (Pareto number 43), which are also highlighted in Tables II and III. As it can be seen in these 
tables, there are 50 responses for the problem. All responses find the installation place of SVC between buses 25 to 29 with different sizes. $70 \%$ of all found responses specify the installation place of SVC at bus 25 , and also $18 \%$ at bus $26,8 \%$ at bus 27 , and $4 \%$ at bus 29 . It can be seen in these tables that the obtained optimal installation place of SVC varies upon different objective functions. For example, the best place for the objective function involving transient stability is bus 25 while for the one involving voltage deviation is buses 26 and 29 . The best installation place of SVC for the total cost objective function is bus 27. Also, Table III indicates the comparison of the cost of power losses in two modes: considering three load levels and one load level. This table shows that with considering three load levels, the power losses in power system are significantly reduced.

\section{TABLE II}

NON -DOMINATED SOLUTIONS ACQUIRED By MEANS OF MOPSO (OPTIMAl Position, Size, AND CONTROLler PARAMETERS OF SVC, SVC COST AND THE FIRST OBJECTIVE).

\begin{tabular}{|c|c|c|c|c|c|c|c|}
\hline 胥总 & $\check{z}$ & $\underset{\infty}{\Xi}$ & 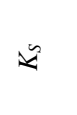 & $\stackrel{2}{*}$ & $\omega^{\infty}$ & 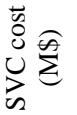 & $\sum_{\bar{l}}^{\infty}$ \\
\hline 1 & 25 & 3.395 & 192.66 & 1.0000 & 0.010 & 3.042 & 60.663 \\
\hline 2 & 25 & 3.693 & 196.21 & 0.9701 & 0.107 & 3.074 & 60.840 \\
\hline 3 & 25 & 3.778 & 200.00 & 0.8535 & 0.134 & 3.113 & 61.057 \\
\hline 4 & 26 & 0.327 & 199.12 & 1.0000 & 0.076 & 0.682 & 55.103 \\
\hline 5 & 25 & 2.170 & 200.00 & 0.9516 & 0.010 & 2.677 & 58.973 \\
\hline 6 & 25 & 0.404 & 196.27 & 1.0000 & 0.010 & 0.858 & 55.306 \\
\hline 7 & 29 & 1.580 & 198.43 & 0.7956 & 0.064 & 2.373 & 58.041 \\
\hline 8 & 29 & 1.458 & 200.00 & 0.9928 & 0.198 & 2.339 & 57.955 \\
\hline 9 & 25 & 1.810 & 197.11 & 1.0000 & 0.010 & 2.489 & 58.358 \\
\hline 10 & 25 & 1.665 & 200.00 & 0.8853 & 0.108 & 2.439 & 58.216 \\
\hline 11 & 26 & 0.971 & 199.01 & 0.8161 & 0.010 & 1.573 & 56.478 \\
\hline 12 & 25 & 0.470 & 198.12 & 1.0000 & 0.154 & 0.892 & 55.519 \\
\hline 13 & 26 & 0.498 & 195.12 & 0.1207 & 0.010 & 0.893 & 55.521 \\
\hline 14 & 26 & 1.022 & 200.00 & 1.0000 & 0.010 & 2.064 & 57.341 \\
\hline 15 & 26 & 0.257 & 198.98 & 0.0382 & 0.977 & 0.491 & 54.932 \\
\hline 16 & 25 & 0.532 & 200.00 & 1.0000 & 0.320 & 1.056 & 55.734 \\
\hline 17 & 25 & 3.259 & 199.10 & 1.0000 & 0.295 & 3.039 & 60.651 \\
\hline 18 & 25 & 2.431 & 197.53 & 0.4083 & 1.000 & 2.876 & 59.810 \\
\hline 19 & 26 & 0.215 & 196.23 & 0.9361 & 0.010 & 0.380 & 54.822 \\
\hline 20 & 25 & 1.812 & 200.00 & 0.5487 & 0.010 & 2.519 & 58.447 \\
\hline 21 & 25 & 3.238 & 200.00 & 1.0000 & 0.010 & 3.013 & 60.509 \\
\hline 22 & 25 & 3.816 & 195.64 & 0.2050 & 0.924 & 3.221 & 61.668 \\
\hline 23 & 25 & 3.696 & 198.03 & 1.0000 & 0.010 & 3.093 & 60.947 \\
\hline 24 & 25 & 3.202 & 200.00 & 0.1030 & 0.939 & 2.999 & 60.433 \\
\hline 25 & 25 & 4.000 & 198.23 & 1.0000 & 0.010 & 3.251 & 61.835 \\
\hline 26 & 25 & 2.258 & 194.45 & 0.0100 & 1.000 & 2.684 & 58.998 \\
\hline 27 & 25 & 3.807 & 200.00 & 1.0000 & 0.103 & 3.167 & 61.366 \\
\hline 28 & 25 & 4.000 & 200.00 & 0.9127 & 0.010 & 3.251 & 61.835 \\
\hline 29 & 25 & 3.049 & 196.12 & 0.4960 & 0.364 & 2.964 & 60.250 \\
\hline 30 & 25 & 0.010 & 199.54 & 0.3070 & 0.514 & 0.019 & 54.473 \\
\hline 31 & 27 & 0.010 & 161.13 & 1.0000 & 0.010 & 0.019 & 54.476 \\
\hline 32 & 25 & 1.969 & 198.21 & 1.0000 & 0.643 & 2.641 & 58.842 \\
\hline 33 & 25 & 0.738 & 200.00 & 0.7242 & 0.010 & 1.549 & 56.081 \\
\hline 34 & 25 & 1.238 & 196.98 & 1.0000 & 0.675 & 2.237 & 57.712 \\
\hline 35 & 25 & 2.927 & 198.33 & 0.8209 & 0.010 & 2.877 & 59.815 \\
\hline 36 & 25 & 2.311 & 200.00 & 0.7276 & 0.180 & 2.761 & 59.298 \\
\hline 37 & 27 & 0.251 & 150.12 & 1.0000 & 0.010 & 0.486 & 55.016 \\
\hline 38 & 25 & 0.992 & 200.00 & 1.0000 & 0.098 & 2.025 & 57.264 \\
\hline 39 & 25 & 1.197 & 198.19 & 1.0000 & 0.010 & 2.132 & 57.481 \\
\hline 40 & 25 & 3.531 & 199.21 & 1.0000 & 0.010 & 3.042 & 60.667 \\
\hline 41 & 26 & 0.010 & 193.20 & 0.9635 & 0.084 & 0.019 & 54.476 \\
\hline 42 & 26 & 0.585 & 193.12 & 0.6186 & 0.178 & 1.235 & 55.979 \\
\hline 43 & 26 & 0.500 & 197.23 & 1.0000 & 0.028 & 0.901 & 55.530 \\
\hline 44 & 25 & 4.000 & 200.00 & 0.8294 & 0.010 & 3.251 & 61.835 \\
\hline 45 & 25 & 4.000 & 200.00 & 1.0000 & 0.359 & 3.251 & 61.835 \\
\hline 46 & 25 & 3.031 & 196.52 & 1.0000 & 0.010 & 2.884 & 59.847 \\
\hline 47 & 27 & 0.010 & 156.13 & 1.0000 & 0.426 & 0.219 & 54.704 \\
\hline 48 & 27 & 0.713 & 155.78 & 0.9589 & 0.103 & 1.411 & 56.232 \\
\hline 49 & 25 & 1.007 & 198.21 & 0.8122 & 0.344 & 2.028 & 57.270 \\
\hline 50 & 25 & 0.355 & 199.32 & 0.8788 & 0.387 & 0.696 & 55.138 \\
\hline
\end{tabular}

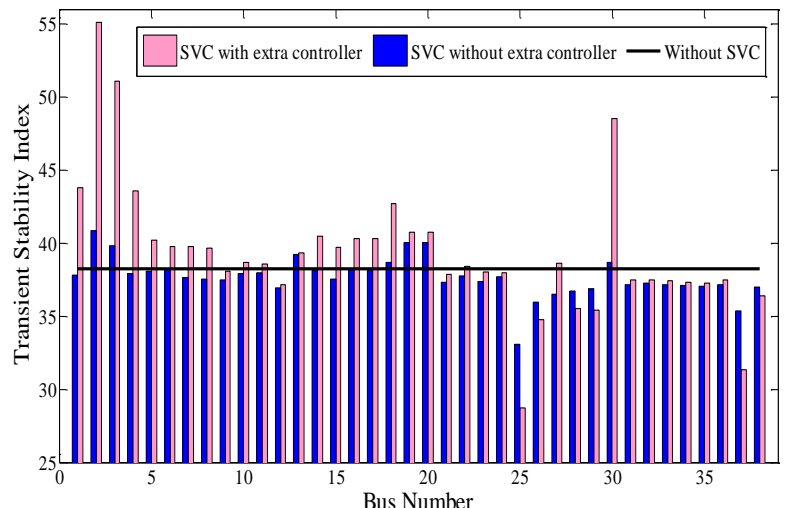

Fig. 3 Transient Stability index of the system for all buses.

Fig. 3 displays the comparison of the transient stability objective over the SVC locations at the entire buses by using the values of the $43^{\text {rd }}$ Pareto answer in Tables II and III. In this figure, the black line indicates the transient stability index when there is no SVC. As shown in this figure, the SVC location to attain the minimum transient stability objective is the bus number 25 .

The other significant point is related to the responses with the weak transient stability of power system such as responses with Pareto solution number 31, 37, 47, 48. In these responses, the SVC installation place is at bus 27 , and the gain of SVC controller has lower amount. These values of gain can help the SVC controller not to deteriorate the transient stability of the system. Fig. 4 to Fig. 7 show the speed deviation and the variation of rotor angle deviations of generators 8 and 5 (generator 10 is the reference), respectively. In these figures, the dash line displays the result without SVC, the spotted line indicates the result using SVC without optimized position and the solid line demonstrates the result using SVC with optimized position.

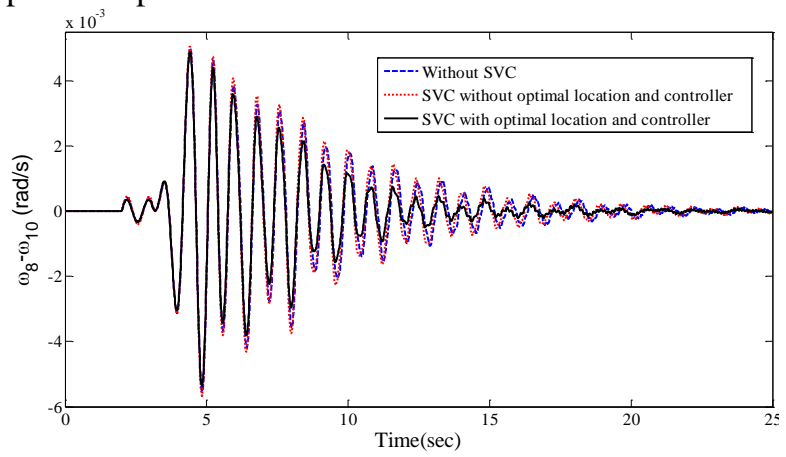

Fig. 4 Generator 8 speed deviation considering both controller and location.

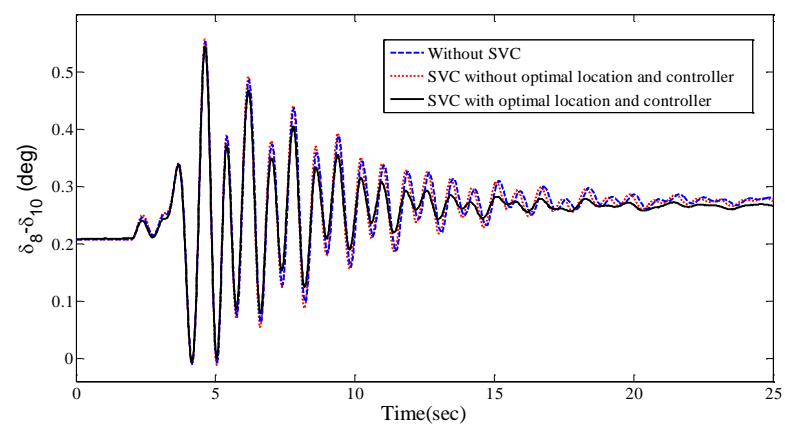


Fig. 5 Generator 8 variation of rotor angle difference considering both controller and location.

Note that, in the case without of the optimized position, the SVC is located at bus 17 , and in the case without enhanced controller, the SVC has no controller and its $V_{\text {ref }}$ is $1 \mathrm{pu}$. These figures verify the results obtained from MOPSO method. Fig. 8 and Fig. 9 show the change of rotor angle deviations and speed deviation of generator 8 for optimal location and size of SVC with and without using the best controller based on the $43^{\text {rd }}$ Pareto solution. It is evident that using SVC with enhanced controller can settle down faster and have more damping.

TABLE III

NON-DOMINATED SOLUTION OBTAINED USING MOPSO (THE SECOND AND THIRD OBJECTIVES VALUES, POWER LOSSES IN THREE INDIVIDUAL LOAD LEVELS AND POWER LOSS COST USING 1 AND 3 LOAD LEVELS)

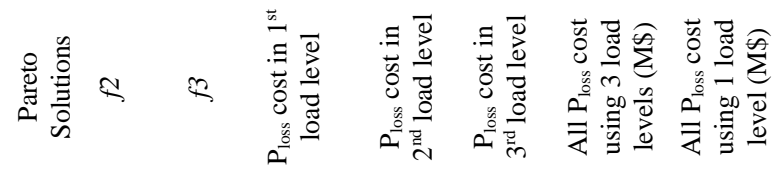

\begin{tabular}{|c|c|c|c|c|c|c|c|}
\hline 1 & 28.13 & 0.0177 & 13.029 & 19.910 & 24.683 & 57.621 & 63.083 \\
\hline 2 & 27.79 & 0.0180 & 13.064 & 19.957 & 24.746 & 57.766 & 63.486 \\
\hline 3 & 27.59 & 0.0182 & 13.107 & 20.015 & 24.823 & 57.944 & 54.444 \\
\hline 4 & 30.43 & 0.0172 & 12.261 & 18.859 & 23.301 & 54.420 & 58.677 \\
\hline 5 & 28.80 & 0.0172 & 12.708 & 19.477 & 24.110 & 56.296 & 60.691 \\
\hline 6 & 30.29 & 0.0177 & 12.268 & 18.866 & 23.312 & 54.447 & 59.002 \\
\hline 7 & 30.47 & 0.0167 & 12.557 & 19.272 & 23.839 & 5.668 & 0.620 \\
\hline 8 & 30.41 & 0.0167 & 12.545 & 19.255 & 23.816 & 55.616 & 50.376 \\
\hline 9 & 29.17 & 0.0170 & 12.606 & 19.338 & 23.926 & 55.869 & 60.889 \\
\hline 10 & 29.19 & 0.0170 & 12.583 & 19.308 & 23.886 & 55.777 & 60.314 \\
\hline 11 & 30.23 & 0.0172 & 12.374 & 19.021 & 23.510 & 54.904 & 59.608 \\
\hline 12 & 30.18 & 0.0177 & 12.309 & 18.928 & 23.390 & 54.627 & 58.886 \\
\hline 13 & 30.50 & 0.0167 & 12.309 & 18. & 23.390 & 54.627 & 58.981 \\
\hline 14 & 30.18 & & 12.463 & & 23.670 & 55.277 & 59.882 \\
\hline 15 & 30.45 & 0.0173 & 12.266 & 18.865 & 23.310 & 54.440 & 58.628 \\
\hline 16 & 30.06 & 0.0177 & 12.321 & 18.945 & 23.412 & 54.678 & 59.140 \\
\hline 17 & 28.01 & 0.0177 & 13.026 & 19.907 & 24.678 & 57.611 & 62.999 \\
\hline 18 & 28.69 & $0.01^{\prime}$ & 12.862 & 19.686 & 24. & 56.933 & 61.751 \\
\hline 19 & 30.56 & 0.01 & 12.266 & 18.865 & 23.3 & 54.442 & 58.386 \\
\hline 20 & 29.07 & & 12.620 & & 23. & 5.928 & 0.465 \\
\hline 21 & 27.99 & & 12.998 & & 24. & 57.495 & 62.951 \\
\hline 22 & 27.71 & 0.0183 & 13.228 & & 25.040 & 58.446 & 64.685 \\
\hline 23 & 27.73 & 0.0180 & 13.084 & & 24.783 & 57.853 & 63.671 \\
\hline 24 & 28.01 & 0.0177 & 12.983 & 19.848 & 24.602 & 57.433 & 62.922 \\
\hline 25 & 27.49 & 0.01 & 13.261 & 20.223 & 25.099 & 58.583 & 65.129 \\
\hline 26 & 28.93 & 0.0 & 12.713 & & 24.118 & 56.314 & 0.749 \\
\hline 27 & 27.57 & & 13.168 & & 24. & 58.198 & 4.639 \\
\hline 28 & 27.42 & & 13.261 & 20. & 25.099 & 58.583 & 65.567 \\
\hline 29 & 28.26 & & 12.947 & & 24.5 & 57.285 & 62.801 \\
\hline 30 & 30.50 & 0.0180 & 12.269 & 18.8 & 23.3 & 54.4 & 58.386 \\
\hline 31 & 31.84 & & 12.270 & & & 54. & 58.386 \\
\hline 32 & 29.01 & & 12.685 & & 24. & 56.201 & 60.643 \\
\hline 33 & 29.91 & & 12.290 & & 23.3 & 54.531 & 59.584 \\
\hline 34 & 29.61 & 0173 & 12.511 & 19.208 & 23.7 & 5.474 & 60.304 \\
\hline 35 & 28.29 & 0.0175 & 12.863 & 19.6 & 24.3 & 56.937 & 61.924 \\
\hline 36 & 28.68 & & 12.766 & 19.556 & 24.214 & 56.537 & 61.698 \\
\hline 37 & 32.16 & 0.0170 & 12.286 & 18.895 & 23.348 & 54.530 & 58.477 \\
\hline 38 & 29.71 & & 12.454 & & 23.654 & 55.239 & 59.627 \\
\hline 39 & & & & 19.167 & 23.7 & 55.349 & 60.271 \\
\hline 40 & 27.80 & & 13.029 & 19.911 & 24.684 & 57.625 & 63.220 \\
\hline 41 & 30.72 & 0.0180 & 12.270 & 18.870 & 23.3 & 54.457 & 58.386 \\
\hline 42 & 30.55 & 0.0168 & 12.336 & 18.967 & 23.440 & 54.744 & 59.050 \\
\hline 43 & 30.43 & 0.0167 & 12.309 & 18.929 & 23.391 & 54.629 & 59.007 \\
\hline 44 & 27.42 & 0.0191 & 13.261 & 20.223 & 25.099 & 58.583 & 65.806 \\
\hline 45 & 27.42 & & 13.261 & & 25.099 & 58.583 & 65.806 \\
\hline 46 & 28.27 & & 12.869 & & & 56.962 & 62.475 \\
\hline 47 & 32.02 & 0.0179 & 12.276 & 18.880 & 23.329 & 54.484 & 58.390 \\
\hline 48 & 31.84 & 0.0173 & 12.355 & 18.993 & 23.473 & 54.821 & 59.281 \\
\hline 49 & 29.75 & 0.0174 & 12.455 & 19.132 & 23.655 & 55.242 & 59.779 \\
\hline 50 & 30.24 & 0.0178 & 12.267 & 18.865 & 23.310 & 54.442 & 58.813 \\
\hline
\end{tabular}

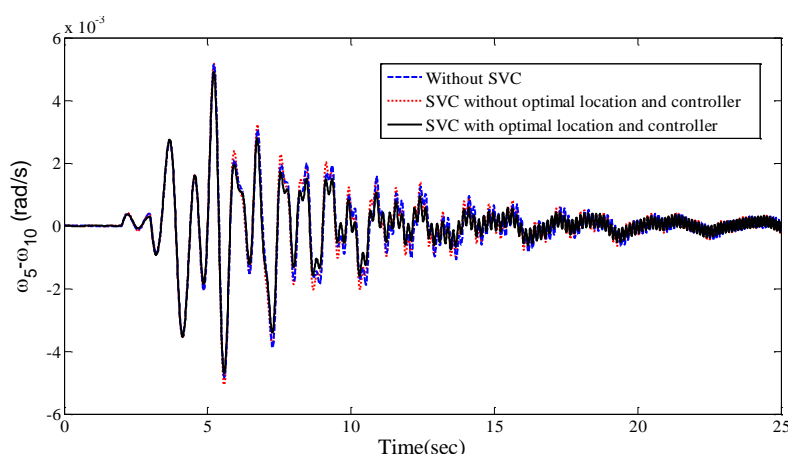

Fig. 6 Generator 5 speed deviation considering both controller and location.

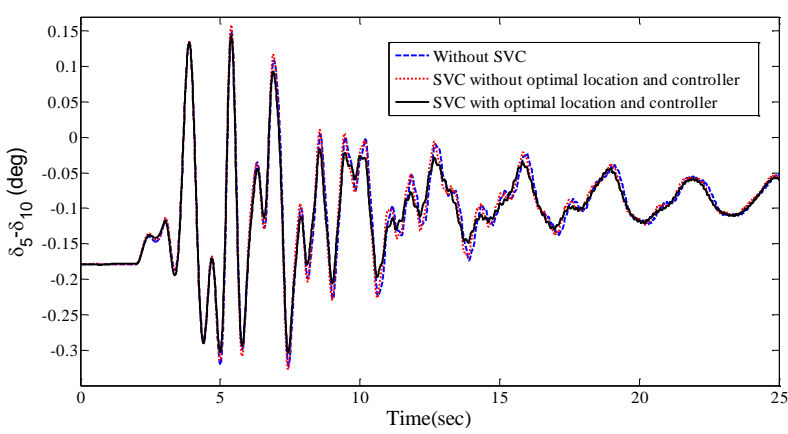

Fig. 7 Generator 5 variation of rotor angle difference considering both controller and location.

Fig. 10 indicates the voltage profile for the $2^{\text {nd }}$ load level. This figure has three response forms: the response without SVC installation, the best and the worst voltage deviation responses. As it can be seen from this figure, even in the worst voltage deviation response, most bus voltages have been improved; but due to bus voltage limits, they are not noticeable.

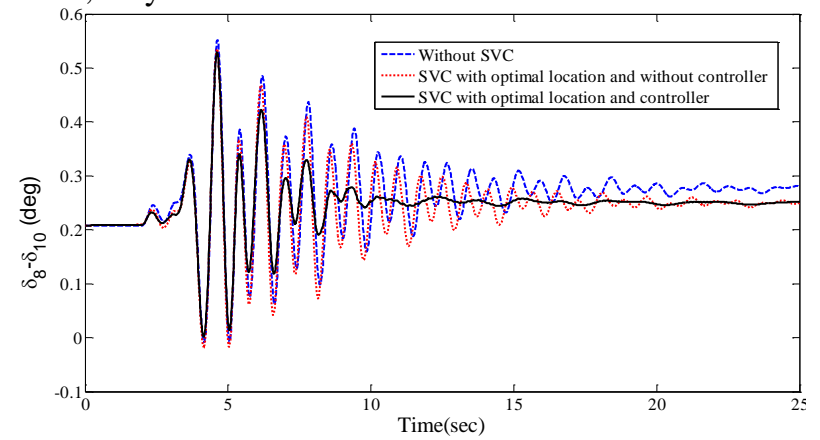

Fig. 8 Generator 8 variation of rotor angle difference considering both only controller.

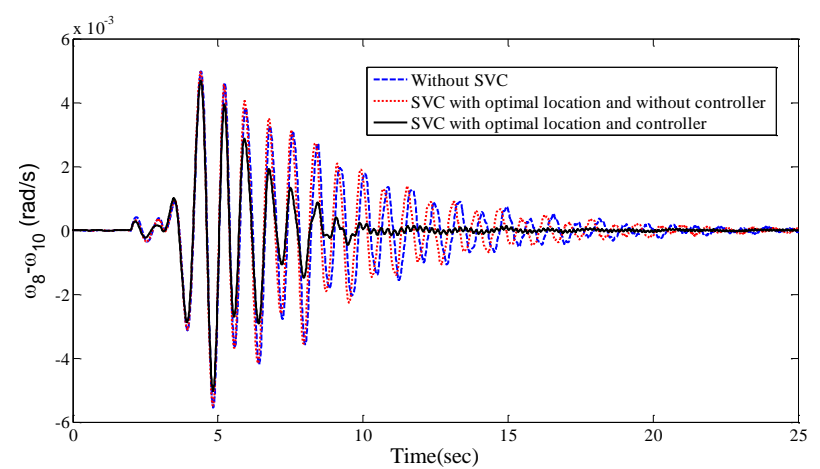

Fig. 9 Generator 8 speed deviation considering both only controller. 


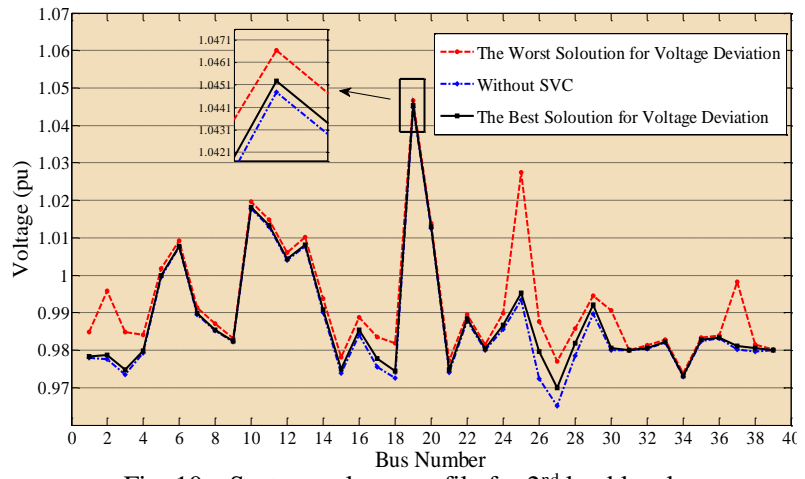

Fig. 10 System voltage profile for $2^{\text {nd }}$ load level.

Another point is related to bus 19 as indicated in the enlarged insertion in Fig. 10. At this bus, the voltage is significantly increased by using the transformer tap value of 1.06. The MOPSO algorithm tries to find the responses which have no increased voltage at this bus. This bus has no electrical load.

\section{CONCLUSION}

In this study, the MOPSO has been utilized as a multi objective optimization technique to define the optimum position, size and parameter setting of SVC in a system with multiple machines. In this research, four objectives have been considered to improve both the static and dynamic conditions. The combination of the active power loss cost and SVC investment cost has been considered as an objective to reach an accurate and practical solution. Improvement of the transient stability and voltage profile of the system have been considered as two separate objectives. Also, an additional controller has been utilized and improved to enhance the performance of SVC in refining the power system transient stability. A 10machine 39-bus New England test system has been utilized to validate the efficacy of suggested MOPSOoptimized size, position and controller parameter setting of the SVC. The nonlinear simulations have revealed that the suggested size, position and controller parameter setting of SVC are different in dynamic and static conditions.

\section{REFERENCES}

[1] N. G. Hingorani, L. Gyugyi, and M. El-Hawary, Understanding FACTS: concepts and technology of flexible AC transmission systems vol. 1: IEEE press New York, 2000.

[2] S. Gerbex, R. Cherkaoui, and A. J. Germond, "Optimal location of multi-type FACTS devices in a power system by means of genetic algorithms," Power Systems, IEEE Transactions on, vol. 16, pp. 537-544, 2001.

[3] Y. Lu and A. Abur, "Static security enhancement via optimal utilization of thyristor-controlled series capacitors," Power Systems, IEEE Transactions on, vol. 17, pp. 324-329, 2002.

[4] S. Singh and A. David, "Congestion management by optimising FACTS device location," in Electric Utility Deregulation and Restructuring and Power Technologies, 2000. Proceedings. DRPT 2000. International Conference on, 2000, pp. 23-28.

[5] D. Mondal, A. Chakrabarti, and A. Sengupta, "Optimal placement and parameter setting of SVC and TCSC using PSO to mitigate small signal stability problem," International Journal of Electrical Power \& Energy Systems, vol. 42, pp. 334-340, 2012.
[6] M. Haque, "Best location of SVC to improve first swing stability limit of a power system," Electric power systems research, vol. 77, pp. 1402-1409, 2007.

[7] M. Gitizadeh and S. Ghavidel, "Improving Transient Stability with Multi-objective Allocation and Parameter Setting of SVC in a Multi-machine Power System," IETE Journal of Research, vol. 60, pp. 33-41, 2014.

[8] M. Gitizadeh, S. Ghavidel, and J. Aghaei, "Using SVC to economically improve transient stability in long transmission lines," IETE Journal of Research, vol. 60, pp. 319-327, 2014.

[9] W. Ongsakul and P. Bhasaputra, "Optimal power flow with FACTS devices by hybrid TS/SA approach," International journal of electrical power \& energy systems, vol. 24, pp. 851-857, 2002.

[10] S.-K. Wang, J.-P. Chiou, and C.-W. Liu, "Parameters tuning of power system stabilizers using improved ant direction hybrid differential evolution," International Journal of Electrical Power \& Energy Systems, vol. 31, pp. 34-42, 2009.

[11] H. E. Talaat, A. Abdennour, and A. A. Al-Sulaiman, "Design and experimental investigation of a decentralized GA-optimized neurofuzzy power system stabilizer," International Journal of Electrical Power \& Energy Systems, vol. 32, pp. 751-759, 2010.

[12] K. Chandrasekaran, S. P. Simon, and N. P. Padhy, "Cuckoo Search Algorithm for Emission Reliable Economic Multi-objective Dispatch Problem," IETE Journal of Research, vol. 60, pp. 128138, 2014.

[13] M. Ghasemi, M. Taghizadeh, S. Ghavidel, J. Aghaei, and A. Abbasian, "Solving optimal reactive power dispatch problem using a novel teaching-learning-based optimization algorithm," Engineering Applications of Artificial Intelligence, vol. 39, pp. $100-108,2015$

[14] M. Ghasemi, M. M. Ghanbarian, S. Ghavidel, S. Rahmani, and E. M. Moghaddam, "Modified teaching learning algorithm and double differential evolution algorithm for optimal reactive power dispatch problem: A comparative study," Information Sciences, vol. 278, pp. 231-249, 2014.

[15] C. A. C. Coello, G. T. Pulido, and M. S. Lechuga, "Handling multiple objectives with particle swarm optimization," Evolutionary Computation, IEEE Transactions on, vol. 8, pp. 256279, 2004.

[16] P. Ngatchou, A. Zarei, and M. El-Sharkawi, "Pareto multi objective optimization," in Intelligent Systems Application to Power Systems, 2005. Proceedings of the 13th International Conference on, 2005, pp. 84-91.

[17] S. Mostaghim and J. Teich, "Covering pareto-optimal fronts by subswarms in multi-objective particle swarm optimization," in Evolutionary Computation, 2004. CEC2004. Congress on, 2004, pp. 1404-1411.

[18] P. Kundur, N. J. Balu, and M. G. Lauby, Power system stability and control vol. 4: McGraw-hill New York, 1994.

[19] A. Zamora-Cárdenas and C. R. Fuerte-Esquivel, "Multi-parameter trajectory sensitivity approach for location of series-connected controllers to enhance power system transient stability," Electric Power Systems Research, vol. 80, pp. 1096-1103, 2010.

[20] Y. Abdel-Magid and M. Abido, "Optimal multiobjective design of robust power system stabilizers using genetic algorithms," Power Systems, IEEE Transactions on, vol. 18, pp. 1125-1132, 2003. 